\title{
Ultraslow Optical Modes in Bose-Einstein Condensates
}

\author{
Özgür E. Müstecaplıoglu ${ }^{a}$ and Devrim Tarhan ${ }^{b}$ \\ ${ }^{a}$ Department of Physics, Koç University, Sarıyer, Istanbul, 34450, Turkey; \\ ${ }^{b}$ Department of Physics, Harran University, Osmanbey Yerleşkesi, Şanlıurfa, Turkey
}

\begin{abstract}
Light can be slowed down to ultraslow speeds $\mathrm{v}$ ia electromagnetically induced transparency in atomic BoseEinstein condensates. This is thought to be useful for storage of quantum information for weak probe pulses. We investigate the effects of inhomogeneous density profile of the condensate on propagation of such ultraslow pulses. We find that spatial density of an atomic condensate leads to a graded refractive index profile, for an off-resonant probe pulse when condensate parameters are suitably chosen. Within the window of negligible absorption, conditions for degenerate multiple waveguide modes are determined. Both analytical and numerical studies are presented to reveal the effects of experimentally controllable parameters, such as temperature and interatomic interaction strength on the number of modes. Group velocity dispersion and modal dispersion are discussed. The effect of waveguide dispersion, in addition to usual material dispersion, on ultraslow pulses is pointed out.
\end{abstract}

Keywords: Bose-Einstein condensates, ultraslow light, waveguiding, dispersion

\section{INTRODUCTION}

At temperatures below the critical temperature of Bose-Einstein condensation, spatial distribution of atoms in optical or magneto-optical traps can be described by a cigar-shaped ellipsoid profile. The ellipsoid consists of a central high-density region, where the condensed atoms lie, surrounded by a low-density thermal gas envelope. In a simple-minded picture, electric susceptibility of the Bose-Einstein condensate (BEC) would be proportional to its density. The refractive index of the central condensate part therefore can be slightly higher than that of the enveloping thermal gas. This situation is then analogous to the case of an optical fiber with a graded-index profile, in which the refractive-index of the central core decreases gradually from center to the core boundary. An important distinction from the fibers is that atomic-condensates are resonant systems while the fibers are nonresonant optical propagation mediums. Exploiting quantum interference ${ }^{1}$ in such atomic resonant medium, remarkable optical pulse propagation effects such as ultraslow light, ${ }^{2-6}$ superluminal light propagation,,$^{7-10}$ stoppage $^{11}$ and storage ${ }^{12,13}$ of optical pulses have been discovered. Most of the theoretical and experimental work has been restricted to one-dimensional situations. Dynamics of optical pulses in two or more dimensions has been investigated very recently numerically. ${ }^{14}$ The possibility of changing the direction of the probe pulse via a control beam has been examined in detail. Other higher dimensional considerations have also been presented. ${ }^{15,16}$ Higher dimensional considerations may prove useful for quantum information processing and storing in addition to manipulation of classical optical pulses.

In this paper we investigate another effect of higher dimensional pulse propagation in a BEC, hinted by our analogy to optical fibers. The refractive index difference between the core and the cladding in a fiber influences the parameter which characterize the number of modes that can be supported by the fiber. In the case of BEC, condensate-thermal gas index difference is negligibly small unless close to an atomic resonance. In order to exploit the high density contrast between the condensate and the thermal gas to allow for multi-mode optical pulses, it is necessary to be close to an atomic resonance. Due to strong absorption nearby the atomic resonance, optical pulse could not propagate much in this case. In previous theoretical and experimental works, pulse propagation under the conditions of electromagnetically induced transparency (EIT) has been utilized to beat the absorption and manipulate the speed of light. However, in this case, the refractive index of the condensate

Further author information: (Send correspondence to Ö.E.M.)

Ö.E.M.: E-mail: omustecap@ku.edu.tr, Telephone: +90 (212) 3381424

Photonic Materials, Devices, and Applications II, edited by Ali Serpengüzel,Gonçal Badenes, Giancarlo Righini

Proc. of SPIE Vol. 6593, 659306, (2007) · 0277-786X/07/\$18 - doi: 10.1117/12.721840

Proc. of SPIE Vol. 6593 659306-1 
remains almost the same with the thermal gas. Hence, under EIT conditions BEC acts as an essentially a singlemode medium. Strictly speaking it can support degenerate two polarization modes only. It has been theoretically shown that such polarization modes can be separated by detuning one of the components slightly away from the EIT resonance, at the cost of some absorption. ${ }^{17}$ Light storage with light of arbitrary polarization has been demonstrated experimentally. ${ }^{18}$

\section{DENSITY PROFILE OF BEC}

At low temperatures a Bose gas can be considered to be composed of two components. The first component becomes the condensate part and the other is a thermal gas background. A simple analytical fitting formula was developed in Ref.. ${ }^{19}$ We will now briefly review this model, where condensate density is evaluated under Thomas-Fermi approximation and the thermal gas density is calculated semi-classically. ${ }^{19,20}$ The total density is then written to be

$$
\rho(\vec{r})=\frac{\mu-V(\vec{r})}{U_{0}} \Theta(\mu-V(\vec{r})) \Theta\left(T_{C}-T\right)+\frac{g_{3 / 2}\left(z e^{-\beta V}\right)}{\lambda_{T}^{3}},
$$

where $U_{0}=4 \pi \hbar^{2} a_{s} / m ; m$ is atomic mass; $a_{s}$ is the atomic s-wave scattering length; $\mu$ is the chemical potential; $\Theta($.$) is the Heaviside step function; g_{n}(x)=\Sigma_{j} x^{j} / j^{n} ; \lambda_{T}$ is the thermal de Bröglie wavelength; $\beta=1 / k_{B} T$; $z=\exp (\beta \mu)$ is the fugacity, and $T_{C}$ is the critical temperature. The external trapping potential is $V(\vec{r})=$ $(m / 2)\left(\omega_{r}^{2} r^{2}+\omega_{z}^{2} z^{2}\right)$ with $\omega_{r}$ the radial trap frequency and $\omega_{z}$ the angular frequency in the $\mathrm{z}$ direction. $\mu$ is determined from $N=\int \mathrm{d}^{3} \vec{r} \rho(\vec{r})$. At temperatures below $T_{c}$ this yields ${ }^{19} \mu(T)=\mu_{T F}\left(N_{0} / N\right)^{2 / 5}$, where $\mu_{T F}$ is the chemical potential evaluated under Thomas-Fermi approximation and the condensate fraction is given by $N_{0} / N=1-x^{3}-s(\zeta(2) / \zeta(3)) x^{2}\left(1-x^{3}\right)^{2 / 5}$, with $x=T / T_{c}$, and $\zeta$ is the Riemann-Zeta function. The scaling parameter $s$ is calculated to be ${ }^{19,21} s=\mu_{T F} / k_{B} T_{C}=\frac{1}{2} \zeta(3)^{1 / 3}\left(15 N^{1 / 6}\left(a_{s} / a_{h}\right)\right)^{2 / 5}$. Here, $a_{h}=\sqrt{\hbar /\left(\omega_{z} \omega_{r}^{2}\right)^{1 / 3}}$ denotes the average harmonic oscillator length scale. At temperatures above the $T_{C}, \mu$ can be determined from $\mathrm{Li}_{3}(z)=\zeta(3) / x^{3}$, where $L i_{3}($.$) is the third-order polylogarithm function. Chemical potential evaluated under$ Thomas-Fermi approximation is defined as $\mu_{T F}=\left(\left(\hbar \omega_{t}\right) / 2\right)\left(15 N a_{s} / a_{h}\right)^{2 / 5}$. Here, $\omega_{t}=\left(\omega_{z} \omega_{r}^{2}\right)^{1 / 3}, N$ is the total atom number and $a_{h}=\sqrt{\hbar /\left(\omega_{z} \omega_{r}^{2}\right)^{1 / 3}}$ denotes the average harmonic oscillator length scale. We have shown the total density as a function of $r$ as seen in the Fig.1.

We assume an anisotropic trapping potential so that the condensate is cigar shaped. Propagation of the probe beam would be along the long axis of the condensate taken to be the $z$-axis. The susceptibility of the medium will be spatially inhomogeneous due to its proportionality to condensate density. Considering cross-sections perpendicular to the $z$-axis, we observe that in the radial direction, the susceptibility of the Bose gas will be analogous to that of a graded index fiber. Inhomogeneity in the propagation direction would be over longer length scale. Thermal gas background here plays the role of the fiber coating while the condensate acts as the core. At lower temperatures compared to $T_{C}$, the index profile approaches to step-index profile. Number density of the medium can be written as the superposition of two contributions by the condensate and the thermal gas background. A simple analytical fitting formula developed in Ref.. ${ }^{19}$ At temperatures well below the $T_{C}$ thermal gas density can be ignored. The radial behavior of the total density at $z=0$ becomes

$$
\rho(r)=\frac{\mu(T)}{U_{0}}\left[1-\left(\frac{r}{R_{T F}}\right)^{2}\right]
$$

\section{SUSCEPTIBILITY OF BEC}

One can calculate susceptibility via local density approximation. We determine the electric susceptibility $\chi$ of BEC consisting of such three-level atoms in EIT scheme and refractive index enhancement scheme. If we obtain electric susceptibility $\chi_{1}$ of an single atom, we can find the susceptibility of the system;

$$
\chi \approx \rho(r) \chi_{1} .
$$




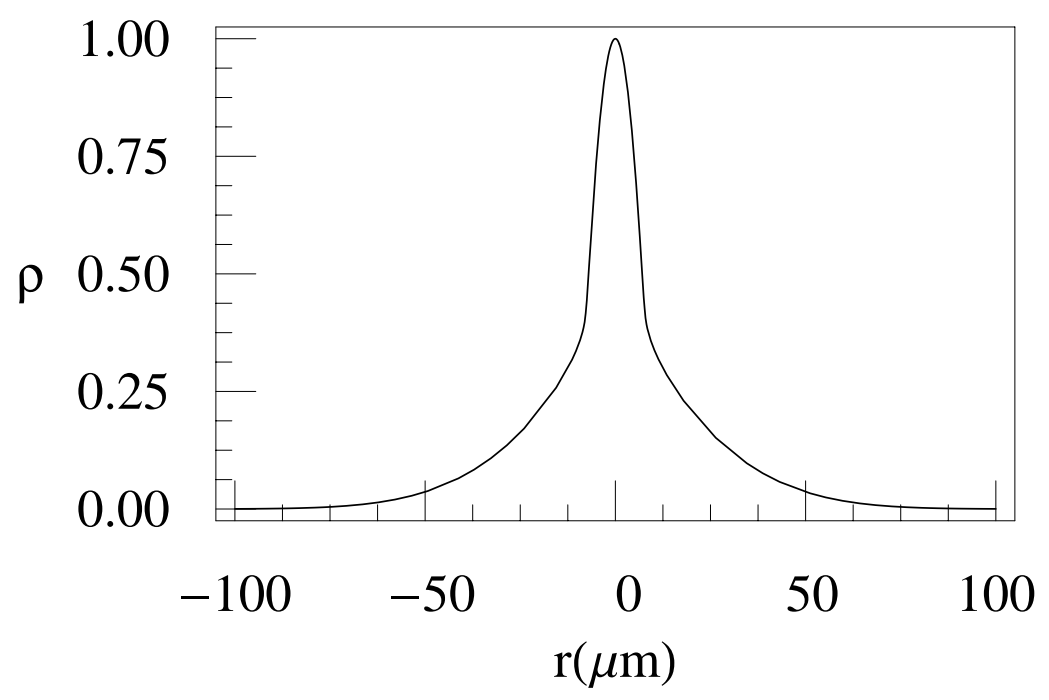

Figure 1. Modelling of the Bose-Condensate as a parabolic function. We have used same parameters; Scattering length, $a_{s}=2.75 \mathrm{~nm}, \lambda=589 \mathrm{~nm}, N=8.3 \times 10^{6}, \omega_{r}=2 \pi \times 69 \mathrm{~Hz}, \omega_{z}=2 \pi \times 21 \mathrm{~Hz}, M=23 \mathrm{amu}$ as for the experiment. The density is normalized by the peak density $3.19 \times 10^{19}\left(1 / \mathrm{m}^{3}\right)$ and $T=42 \mathrm{nK} .^{4}$

We calculate the refractive index by the formula $n=\sqrt{1+\chi}$. If we write the susceptibility again, we will obtain

$$
\chi(r)=\left\{\begin{array}{ll}
A\left\{\left[1-\left(\frac{r}{R_{T F}}\right)^{2}\right]\right\} & r \leq R_{T F} \\
0 & r \geq R_{T F}
\end{array} .\right.
$$

where $R_{T F}$ is the Thomas-Fermi radius which is defined as $\sqrt{2 \mu(T) / m \omega_{r}^{2}}$. Here $A$ depends on susceptibility of the atomic systems. We denote the refractive index, $n(r)$ as

$$
n(r)=\left\{\begin{array}{ll}
n_{1}\left[1-2 \triangle\left(\frac{r}{R_{T F}}\right)^{2}\right]^{1 / 2} & r \leq R_{T F} . \\
n_{1}(1-2 \Delta)^{1 / 2} & r \geq R_{T F}
\end{array} .\right.
$$

where $2 \triangle=\left(n_{1}^{2}-n_{2}^{2}\right) / 2 n_{1}^{2}{ }^{22} \quad$ The refractive index is shown in the Fig.?? for some numerical parameters.

EIT is a technique for making cancellation of induced absorption to weak probe field tuned in resonance to an atomic transition by applying a strong resonant electromagnetic field to couple coherently another atomic transition. ${ }^{1}$ In this scheme, it is assumed that the only dipole forbidden transition is the one between the lower levels. The upper level is coupled to the lower levels via a strong drive field with frequency $\omega_{c}$ and a weak probe field of frequency $\omega_{p}$. Susceptibility $\chi$ for the probe transition can be calculated as a linear response as most of the atoms remain in the lowest state. Assuming local density approximation, neglecting local field, multiple scattering and quantum corrections and employing steady state analysis $\chi$ is found to be ${ }^{23}$

$$
\chi=\frac{\rho\left|\mu_{31}\right|^{2}}{\epsilon_{0} \hbar} \frac{i\left(-i \Delta+\Gamma_{2} / 2\right)}{\left[\left(\Gamma_{2} / 2-i \Delta\right)\left(\Gamma_{3} / 2-i \Delta\right)+\Omega_{c}^{2} / 4\right]},
$$

where $\Delta=\omega_{31}-\omega_{p}$ is the frequency detuning of the probe field with frequency $\omega_{p}$ from the resonant electronic transition $\omega_{31}$. For the cold gases considered in this paper, Doppler shift in the detuning is neglected. It is assumed that the drive field is at resonance. $\Omega_{c}$ is the Rabi frequency of the drive field; $\mu_{31}$ is the dipole matrix element between states $|3\rangle$ and $|1\rangle$ which can also be expressed in terms of resonant wavelength $\lambda_{31}$ of the probe transition via $\mu_{31}^{2}=3 \epsilon_{0} \hbar \lambda_{31}^{3} \gamma / 8 \pi^{2}$ with $\gamma$ is the radiation decay rate between $|3\rangle$ and $|1\rangle . \Gamma_{2}$ and $\Gamma_{3}$ denote the dephasing rates of the atomic coherence between the appropriate states. $\rho$ stands for the atomic density. 


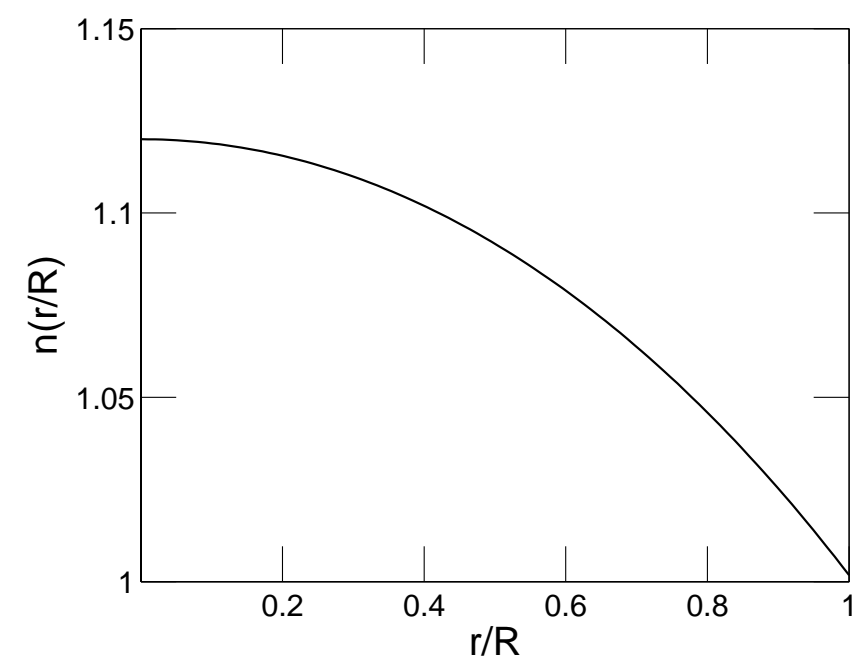

Figure 2. Index of refraction of the core is displayed for some numerical values such as $n_{1}=\sqrt{1+A}=1.12$ and $n_{2}=1$. All parameters are the same as in Fig.(1).

\section{LIGHT PROPAGATION AND MODE DISPERSION}

We use the cylindrical coordinate system because the refractive index profile $n(r)$ is cylindrically symmetric. If we take transverse component of the linearly polarized electric field of the (LP) modes as in Ref. ${ }^{22}$

$$
E_{t}=\psi(r) e^{i l \phi} \exp \left[i\left(\omega t-\beta_{l m} z\right)\right]
$$

Here $l, m=0,1,2,3, \ldots, \phi$ is the absolute phase and $\beta_{l m}$ is the propagation constant in the $z$ direction. The wave equation for the field vector field $E_{t}$ is:

$$
\left[\nabla^{2}+k^{2}\right] E_{t}=0 .
$$

where $k^{2}=\omega^{2} n^{2} / c^{2}$ and $\nabla^{2}$ is the Laplacian operator in the cylindrical coordinate. The relationship between angular frequency and wave number (propagation constant in the radial direction) is:

$$
\omega^{2}=\frac{c^{2}}{n^{2}}\left(\beta_{l m}^{2} \mp q^{2}\right) .
$$

Here the radial wave number $q^{2}=k_{x}^{2}+k_{y}^{2}$ and $\beta_{l m}$ is the propagation constant in the $z$ direction. If we put Eq.7 in the Eq.8,we will obtain:

$$
\left[\frac{d^{2}}{d r^{2}}+\frac{1}{r} \frac{d}{d r}+p^{2}(r)\right] \psi(r)=0,
$$

in which $p^{2}(r)=k_{0}^{2} n^{2}(r)-\beta_{l m}^{2}-l^{2} / r^{2}{ }^{22}$ Since the refractive index does not vary appreciably over the distance on the order of one wavelength, WKB approximation may be used for solving the graded-index BEC. ${ }^{22}$ In this approximation $\psi(r)$ is generally taken in the following form in order to solve Eq. (10) $\psi(r)=e^{i S(r)}$, where $S(r)$ is a complex function of $r$. Applying the Bohr-Sommerfeld quantization rule to the $p(r),{ }^{26}$ the mode becomes

$$
\int_{r_{1}}^{r_{2}}\left[k_{0}^{2} n^{2}(r)-\beta_{l m}^{2}-\frac{l^{2}}{r^{2}}\right]^{1 / 2} d r=\left(m+\frac{1}{2}\right) \pi
$$

Here $k_{0}=2 \pi / \lambda$ and $m=0,1,2,3, \ldots$. The mode condition can be solved analytically for the propagation constant $\beta_{l m}$. If we solve Eq.(11),we obtain the propagation constant $\beta_{l m}$ which is defined as ${ }^{22}$

$$
\beta_{l m}=n_{1} k_{0}\left[1-\frac{2(2 \triangle)^{1 / 2}}{n_{1} k_{0} R_{T F}} K\right]^{1 / 2}
$$


where $K=(|l|+2 m+1)$. The propagation constant $\beta_{l m}$, depends on the frequency of the light. This frequency dependence determines the dispersion of the waveguide. Optical pulse waves can be dispersive because of the material used as the graded-index fiber. Propagation constant is defined in terms of mode index $\beta_{l m}=n_{l m} k_{0}=$ $n_{l m}\left(n_{1}, n_{2}, \omega\right) \omega / c$. The group velocity $v_{g}$ is defined as

$$
\frac{1}{v_{g}}=\frac{d \beta}{d \omega}=(\omega / c)\left[\frac{\partial n_{l m}}{\partial n_{1}} \frac{\partial n_{1}}{\partial \omega}+\frac{\partial n_{l m}}{\partial n_{2}} \frac{\partial n_{2}}{\partial \omega}+\frac{\partial n_{l m}}{\partial \omega}\right]+n_{l m} / c .
$$

Here, the first two terms in the square bracket are the contribution from material dispersion, the third term is waveguide dispersion. ${ }^{22}$ Therefore the group velocity is characterized by

$$
v_{g}=\frac{c}{n_{l m}+\omega \frac{\partial n_{l m}}{\partial n_{1}} \frac{\partial n_{1}}{\partial \omega}+\omega \frac{\partial n_{l m}}{\partial \omega}} .
$$

Here $c$ is the speed of the light in the free space.

\section{RESULTS AND DISCUSSIONS}

\subsection{Conditions for single-mode and multi-mode wave propagation through a Bose-Einstein condensate}

Under EIT conditions, in Eq.14 when we compare waveguide dispersion with the material dispersion, material dispersion is much bigger than the waveguide dispersion, therefore we can neglect waveguide dispersion term. Mode index $n_{l m}$ is equal to 1.01968 under EIT scheme, so we can take it as 1. Above existing circumstances, the group velocity becomes,

$$
v_{g}=\frac{c}{1+\omega \frac{\partial n_{l m}}{\partial n_{1}} \frac{\partial n_{1}}{\partial \omega}}
$$

We illustrate the group velocity as a function of $K$ at the temperature $T=42 \mathrm{nK}$ in Fig.??. We calculate the group velocity for the eit condition under some numerical parameters; $n_{1}=1.01968,2 \triangle=0.2, R_{T F}=21.1 \mu \mathrm{m}$ and $k_{0}=1.07 \times 10^{7}(1 / \mathrm{m})$. The group velocity is $v_{g}=29.9 \mathrm{~m} / \mathrm{sec}$ for the single mode under eit condition. As seen in the Fig.?? group velocity increases with the mode number $K$ which is defined as $(|l|+2 m+1)$. The normalized frequency which determines the maximum mode condition is computed from definition $V=45$.

Under EIT conditions, if we compare imaginary and real parts of the susceptibility;imaginary part $\chi^{\prime \prime}$ of $\chi$ is very small. We consider a gas of $N=8.3 \times 10^{6}{ }^{23} \mathrm{Na}$ atoms for which $M=23 \mathrm{amu}, \lambda_{0}=589 \mathrm{~nm}, \gamma=2 \pi \times 10.01$ $\mathrm{MHz}, \Gamma_{3}=0.5 \gamma, \Gamma_{2}=2 \pi \times 10^{3} \mathrm{~Hz}$, and $a_{s}=2.75 \mathrm{~nm}$. For the parameters of the trapping potential, we take $\omega_{r}=2 \pi \times 69 \mathrm{~Hz}$ and $\omega_{z}=2 \pi \times 21 \mathrm{~Hz}$ as in Ref.. ${ }^{4}$ The coupling field Rabi frequency is taken to be $\Omega_{c}=2.5 \gamma$. For these parameters we obtain $\chi^{\prime}=0.04$ and $\chi^{\prime \prime}=0.0006$. Detuning parameter is obtained for the single mode condition, $\Delta=0.1 \gamma$ where $\gamma=6.29 \times 10^{7} 1 /$ sec. We have modelled Eq. 1 and we obtained peak density for the atomic density which is denoted as $\rho_{0}=1.59 \times 10^{20}\left(1 / \mathrm{m}^{3}\right)$. The normalized frequency $V$ is defined as $k_{0} R_{T F}\left(n_{0}^{2}-n_{2}^{2}\right)^{1 / 2}$. The parameter $V$ determines the number of mode supported by the fiber.The refractive index is defined as $n_{1}=(1+\chi)^{1 / 2}$.

In practice the parabolic-index profile is truncated at the core boundary $r=R_{T F}$. We find a confined mode description for the propagation constant, $\beta, k_{0}<\beta<n_{0} k_{0}$. The subscription is

$$
2(|l|+2 m+1) \leq k_{0} R_{T F} \sqrt{A}=V
$$

where $V=k_{0} R_{T F}\left(n_{1}^{2}-n_{2}^{2}\right)^{1 / 2}, A=3 \lambda^{3} \mu(T) / 40 \pi^{2} U_{0}$, for $r>R_{T F} n_{2}$ is the clad refractive index, $n_{2}=$ $n_{0}(1-2 \triangle)^{1 / 2}$ or $n_{2}=1$.

The lowest value of the $k_{0} R_{T F} \sqrt{A}$ is 9.2 at temperature $428.4 \mathrm{nK}$. 


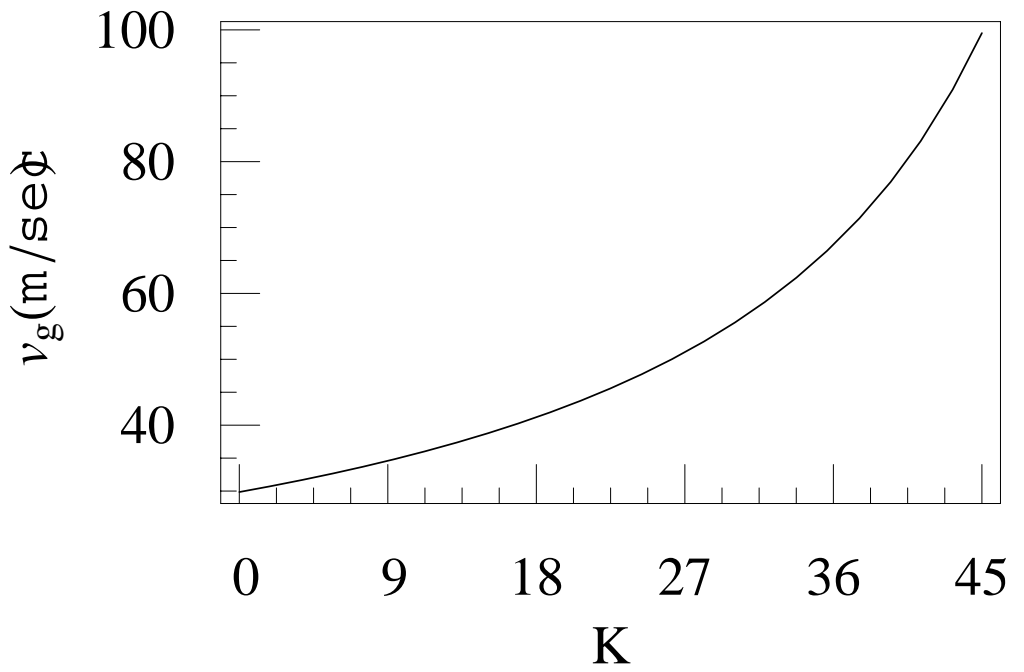

Figure 3. Dependence of the group velocity on $K=(|l|+2 m+1)$ at $T=42 \mathrm{nK}$. For the single mode condition $(K=0$ ) group velocity is $29.9(\mathrm{~m} / \mathrm{sec})$.

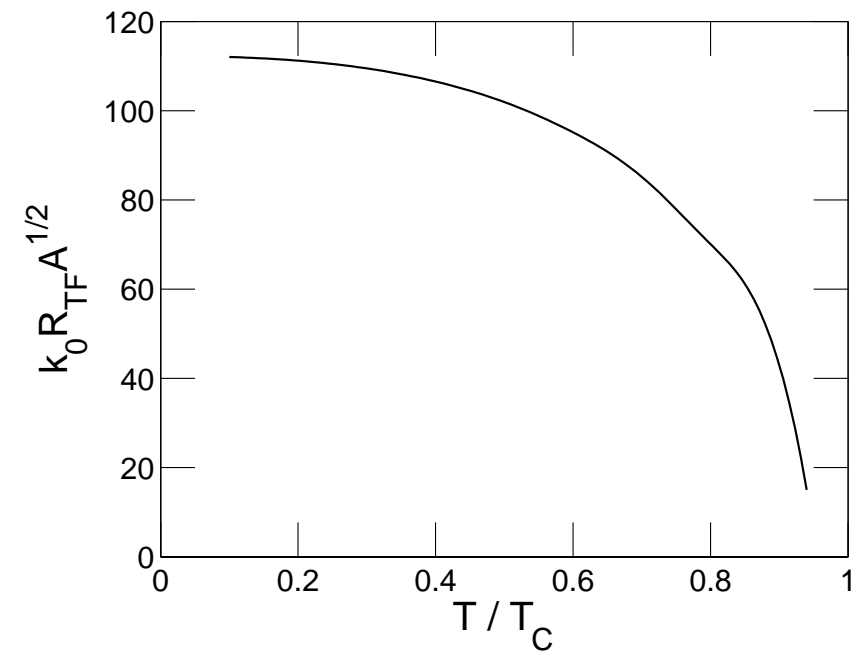

Figure 4. Temperature dependent of $k_{0} R_{T F} \sqrt{A}$. Parameters used are: Scattering length, $a_{s}=2.75 \mathrm{~nm}, \lambda=589 \mathrm{~nm}$, $N=8.3 \times 10^{6}, \omega_{r}=2 \pi \times 69 \mathrm{~Hz}, \omega_{z}=2 \pi \times 21 \mathrm{~Hz}, M=23 \mathrm{amu}$ as for the experiment. ${ }^{4}$ 


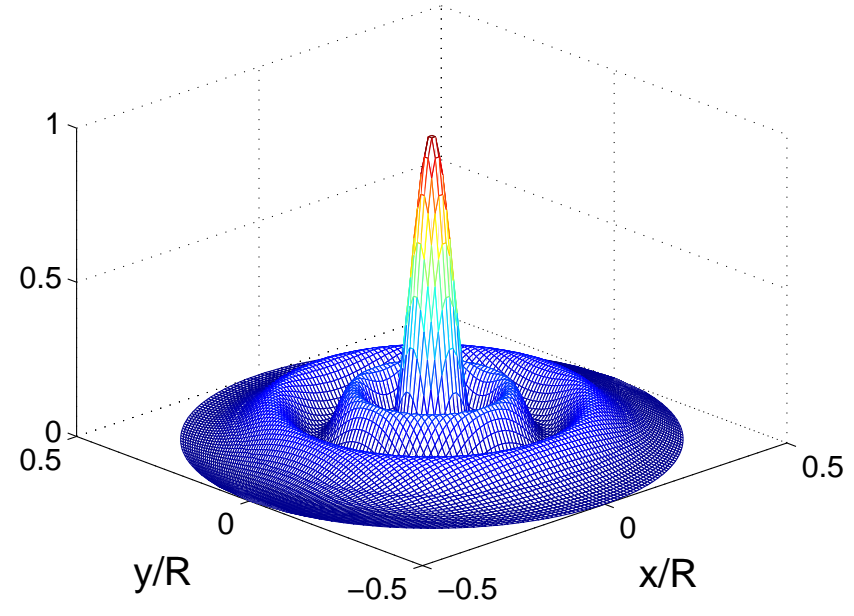

Figure 5. $\mathrm{T}\left|\psi_{00}\right|^{2}$ is displayed and $L P_{00}$ is proportional with $\psi_{00} . x$ and $y$ are normalized by $R=3 \mu \mathrm{m}$.

Table 1. Propagation constants obtained by WKB method

\begin{tabular}{|c|c|c|c|c|}
\hline & $\beta_{1} R$ & $\beta_{2} R$ & $\beta_{3} R$ & $\beta_{4} R$ \\
\hline$l=0$ & 32.4740 & 33.4395 & 34.3779 & 35.2914 \\
\hline$l=1$ & 31.9803 & 32.9603 & 33.9119 & 34.8376 \\
\hline
\end{tabular}

\subsection{Mode Patterns}

More accurate results may be obtained using a numerical scheme. For that purpose it is assumed that the index of refraction increases incrementally from the edge to the center of the core. If the number of increments is high enough, this model will be a faithful representation of the conditions prevailing in the inhomogeneous core. If the turning point is in the interval $\mathrm{m}$ then the solution of the wave equation in the interval $i<k$ will be $\psi=A_{i} J_{l}\left(q_{i} r\right)+B_{i} Y_{l}\left(q_{i} r\right)$. Where $J_{l}, Y_{l}$ are Bessel functions of the first and second kind of order $l$. $q_{i}$ is $\sqrt{\omega^{2} n_{i}^{2} / c^{2}-\beta^{2}}$. $n_{i}$ is the index of refraction in the interval $i$. For $i>k$, the index of refraction is lower than that of the turning point and accordingly the solution of the wave equation becomes $\psi=A_{i} I_{l}\left(q_{i} r\right)+B_{i} K_{l}\left(q_{i} r\right)$ where $I_{l}, K_{l}$ are modified Bessel functions of the first and second kind. In that case $q_{i}$ is $\sqrt{-\omega^{2} n_{i}^{2} / c^{2}+\beta^{2}}$. The boundary conditions are $B_{0}=0$ (The wave is bounded at the origin) and $A_{N+1}=0$ (the wave does not diverge at infinity.) Electromagnetic boundary conditions for the graded index media re1quires the function $\psi$ and its radial derivative to be continuous. This continuity provides a recurrence relation between successive coefficients $A_{i}, B_{i}$. For $i<k$ the recurrence relation is more stable for increasing $m$. The coefficients $A_{k}$ and $B_{k}$ at the turning point may be calculated from $A_{0}$ or alternatively they may be derived from $B_{N}+1$. In both derivations the ratio $A_{k} / B_{k}$ must have the same value. The preservation of this ratio provides an equation for the wave number $\beta$. Our typical results are given in Fig. 5 and Fig. 6. A comparison of WKB method and the numerical treatment is presented in Tables (2) and (1).

Table 2. Numerically evaluated propagation constants

\begin{tabular}{|c|c|c|c|c|}
\hline & $\beta_{1} R$ & $\beta_{2} R$ & $\beta_{3} R$ & $\beta_{4} R$ \\
\hline$l=0$ & 32.4914 & 33.4400 & 34.3778 & 35.2912 \\
\hline$l=1$ & 32.0661 & 32.9635 & 33.9119 & 34.8375 \\
\hline
\end{tabular}




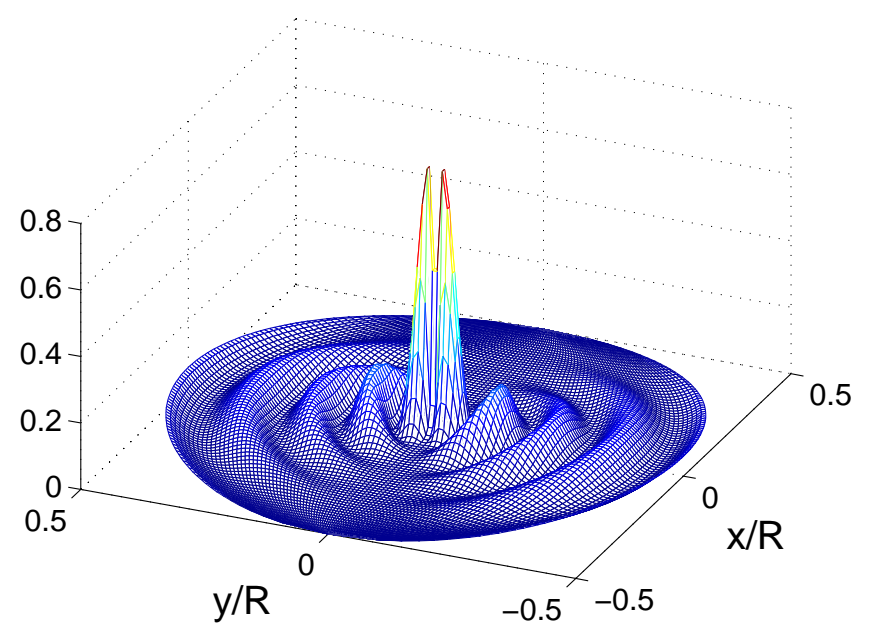

Figure 6. $\left|\psi_{10}\right|^{2}$ is displayed and $L P_{10}$ is proportional with $\psi_{10} . x$ and $y$ are normalized by $R=3 \mu \mathrm{m}$.

\section{CONCLUSION}

We have modelled Bose-Einstein condensate as a graded-index fiber. We have discussed light modes of the Bose-Einstein condensate in the graded index fiber. We find the propagation constant $\beta_{l m}$ in the z direction by WKB approximation and by numerical methods. Also we find mode subscription which is shown in the Eq.16. Radiuses for the single and two mode condition are analytically calculated. We have examined optical mod numbers that can be supported by atomic Bose-Einstein condensate(BEC). We have shown that, the number of modes supported by the fiber depends on temperature. Under EIT conditions BEC acts as an essentially a single-mode medium. But many modes whose group velocities are very small have been obtained with acceptable absorption. Using EIT, we have examined group velocity. It depends on mode number $K$.

Moreover, we have numerically examined optic modes and propagation constant in the z direction in the graded-index fiber. Our results show that propagation constant $\beta$ is approximately same as that predicted by the analytically calculations with WKB approximation. For optical communication purposes graded-index profile is important due to its mode dispersion properties. In order to enhance quantum information and storage, three dimensional considerations may helpful.

\section{ACKNOWLEDGMENTS}

Ö.E.M. acknowledges support by Turkish Academy of Sciences under Young Scientist Program Grant TÜBAGEBİP and by FP6-IST 511616 Network of Excellence PHOREMOST. We acknowledge N. Postacioglu for useful discussions and help in numerical computations.

\section{REFERENCES}

1. S. E. Harris "Electromagnetically induced transparency," Physics Today 50, 36-42 (1997).

2. M. M. Kash, V. A. Sautenkov, A. S. Zibrov, L. Hollberg, G. R. Welch, M. D. Lukin, Y. Rostovtsev, E. S. Fry, and M. O. Scully, "Ultraslow Group Velocity and Enhanced Nonlinear Optical Effects in a Coherently Driven Hot Atomic Gas," Phys. Rev. Lett. 82, 52295232 (1999).

3. D. Budker, D. F. Kimball, S. M. Rochester, and V. V. Yashchuk, "Nonlinear Magneto-optics and Reduced Group Velocity of Light in Atomic Vapor with Slow Ground State Relaxation," Phys. Rev. Lett. 83, 17671770 (1999).

4. L.V. Hau, S.E. Harris, Z. Dutton, and C.H. Behroozi, "Light speed reduction to 17 metres per second in an ultracold atomic gas," Nature 397, 594-598 (1999). 
5. G. Morigi and G. Agarwal, "Temperature variation of ultraslow light in a cold gas," Phys. Rev. A 62, 013801 (2000).

6. Müstecaplıõlu Ö E and You L "Slow light propagation in trapped atomic quatum gases," Phys. Rev. A 64, $013604(2001)$

7. A. Dogariu, A. Kuzmich, and L. J. Wang, "Transparent anomalous dispersion and superlminal light-pulse propagation at a negative group velocity," Phys. Rev. A 63, 053806 (2001).

8. L.J. Wang, A. Kuzmich, and A. Dogariu, "Gain-assited superluminal light propagation," Nature 406, 277 (2000).

9. G.S. Agarwal, T.N. Dey, and S. Menon, "Knob for chancing light propagation from subluminal to superluminal," Phys. Rev. A 64, 053809 (2001).

10. M. Artoni, G. La Rocca, F. Cataliotti, and F. Bassani, "Highly anomalous group velocity of light in ultracold rubidium gases," Phys. Rev. A 63, 023805 (2001).

11. O. Kocharovskaya, Y. Rostovtsev, and M.O. Scully, "Stopping light via hot atoms," Phys. Rev. Lett. 86, $628(2001)$.

12. C. Liu, Z. Dutton, C. H. Behroozi, and L. V. Hau, "Observation of coherent optical information storage in an atomic medium using halted light pulses," Nature 409, 490-493 (2001).

13. D. F. Phillips, A. Fleischhauer, A. Mair, R. L. Walsworth, and M. D. Lukin, "Storage of Light in Atomic Vapor," Phys. Rev. Lett. 86, 783786 (2001).

14. P. Arve, P. Jänes, and L. Thylén, "Propagation of two-dimensional pulses in electromagnetically induced transparency media," Phys. Rev. A 69, 063809 (2004).

15. L.-M. Duan, J.I. Cirac, and P. Zoller, "Three-dimensional theory for interaction between atomic ensembles and free-space light," Phys. Rev. A 66, 023818 (2002).

16. Ö. Müstecaplıog̃lu and L. You, "Propagation of raman-matched laser pulses through a Bose-Einstein condensate," Opt. Commun 193, 301-312 (2001).

17. G.S. Agarwal and S. Dasgupta, e-print quant-ph/0201092 (2002).

18. H. Gao, M. Rosenberry, and H. Batelaan, "Light storage with light of arbitrary polarization," Phys. Rev. A 67, 053807 (2003).

19. M. Naraschewski, D. M. Stamper-Kurn, "Analytical description of a trapped semi-ideal Bose gas at finite temperatue," Phys. Rev. A 58, 2423-2426 (1998).

20. V. Bagnato, D.E. Pritchard, D. Kleppner, "Bose-Einstein condensation in an external potential," Phys. Rev. A 35, 4354-4358 (1987).

21. S. Giorgini, L. P. Pitaevskii, S. Stringari, "Thermodynamics of a Trapped Bose-Condensed Gas," J. Low Temp. Phys. 109, 309 (1997).

22. A. Yariv, Optical Electronics, (Saunders College,Holt,Rinehart and Wiston 1991), pp.101.

23. M. O. Scully ve M. S. Zubairy, Quantum Optics, (Cambridge, Cambridge, 1997).

24. M. Bashkansky, G. Beadie, Z. Dutton, F. K. Fatemi, J. Reintjes, and M. Steiner, "Slow-light dynamics of large-bandwidth pulses in warm rubidium vapor," Phys. Rev. A 72, 033819 (2005).

25. M. O. Scully,"Enhancement of the index of refraction via quantum coherence" Phys. Rev. Lett. 67, 1855 (1991).

26. L. D. Landau and E. M. Lifshitz, Quantum Mechanics, (Pergamon Press,1958). 